\title{
Performance Evaluation of Traveling Wave Fault Locator for a 220kV Hoa Khanh-Thanh My Transmission Line
}

\author{
Kim Hung Le \\ The University of Danang, University of Science and \\ Technology, Da Nang, Viet Nam \\ lekimhung@dut.udn.vn
}

\author{
Phan Huan $\mathrm{Vu}$ \\ Central Power Corporation, Center Electrical Testing \\ Company Limited, Da Nang, Viet Nam \\ vuphanhuan@gmail.com
}

\begin{abstract}
This paper presents the traveling wave based fault location methods of SEL-400L, and SFL-2000 available on the market for a $66.9 \mathrm{~km}, 220 \mathrm{kV}$ Hoa Khanh-Thanh My transmission line in Central Viet Nam, such as single-ended, and doubleended, all of which rely on measurements from inductive CTs and capacitive VTs. Focus was given on the building process of a Matlab Simulink model to evaluate these methods. Current and voltage signals were sent to an analog Chebyshev type II filter which passes higher frequency signals at $3 \mathrm{kHz}$ and rejects low frequencies signal at $50 \mathrm{~Hz}$. After that, these output signals are used in Clarke's transformation for getting 0 and $\alpha$ components. The detail coefficient of the selected components after DWT using Db4 wavelet at decomposition level 1 can be used to determine the fault types, the direction of fault and propose a crest-wave comparison solution to identify exactly the adjacent bus' reflected wave from the fault point's reflected wave for the fault location. Finally, the accuracy of fault location on the transmission line is reviewed by varying various parameters like fault type, fault location and fault resistance on a given power system model.
\end{abstract}

Keywords-transmission line; traveling wave fault locator; single ended method; double ended method; Matlab/Simulink

\section{INTRODUCTION}

Numerical relays are the most popular devices used for transmission line protection. They include fault location estimation based on impedance methods, which uses the voltage and current data measured in $50 \mathrm{~Hz}$ at one or more points along the power networks after the occurrence of a fault. Typical impedance method's error ranges from $2 \%$ to $5 \%$ depending on the relay model according to industry standards. However, the actual error is usually larger than $5 \%$ in practice operation manager at EVN of Viet Nam [1]. It can be influenced by weather, high resistance ground faults, measurement errors, line impedance errors, mutual coupling, compensated lines, and other factors [2]. Therefore, finding the accurate location of a fault constitutes a challenge in the power operator. Selecting a new appropriate technology for a fault location application can be a daunting task so a performance evaluation is required when the EVN wants to compare accuracy and finds the optimum and the most cost-effective one. In 2017, EVN's project installed a traveling wave fault locator (TWFL) equipment Kinkei SFL-2000, SEL-400L for $220 \mathrm{kV}, 500 \mathrm{kV}$ transmission lines in substations such as Son Ha, Thanh My, Hoa Khanh, Hue, Dong Ha, Tam Ky, and Doc Soi in central Viet Nam. The project implementation plan followed 3 steps. The first step was setting up the TWFL to work with single-ended method. The second step was synchronizing the TWFLs by the Furuno GPS/GNSS clock receiver and using a configured communication channel. The TWFLs would send the traveling wave arrival information to $\mathrm{F} / \mathrm{L}$ server that calculates the distance to fault base on doubleended method, display results and sends email to the operator. The third step, EVN installs an NPT communication network link between TWFLs as shown in Figure 1. The TWFLs receive the remote traveling wave information that is necessary to provide an automatic fault location. SFL-2000's accuracy is reported to be $200 \mathrm{~m}$ [3], whereas the accuracy for SEL-400L is reported to be $2 \%$ [2].

Although this issue was recognized by PTC2 as a good way to overcome the shortfalls of impedance-based methods, the SFL-2000 is not able to be used in step 1. So, if it is impossible to have communication between ends, the fault location cannot be implemented automatically by the F/L server. Note that, the problem faced by the power system operator is manually collected traveling waves event reports from each substation. They lack skills to discriminate the reflection wave of fault point, the time of arrival of the wave for manual fault location estimation using the single-ended method because the reflected surge from the adjacent bus has the same polarity as the real fault point's reflected wave, so the confusion is inevitable. Almost as many respondents said all the implementation and support work would be done by experienced professionals. Consequently, EVN needs to conduct a training session about the knowledge of the problem of fault location using traveling wave signals for communication technicians, field personnel, relay engineers, and analysis an in-depth review of all TWFL system operations. In order to solve this issue, the paper focuses on TWFL based double-ended and single-ended methods implemented in a $66.9 \mathrm{~km}, 220 \mathrm{kV}$ Hoa Khanh-Thanh My transmission line by Matlab Simulink. The proposed model has been assessed through several scenarios. Results show that methods consistently and significantly yielded the accurate 
location of the actual fault.

\section{TRAVELING WAVE BASED FAULT LOCATION METHODS}

The TWFL can work based on current or voltage signals. It has two kinds of the traveling wave (TW) methods that are used for most fault locator systems. One is the single-ended, which captures data from the initial traveling wave and subsequent reflections at one terminal to calculate the fault location without requiring any information from the relay at the remote terminal. The other is double-ended, which requires data information from two terminals which are both equipped with a GPS receiver to time tag the exact moment the traveling wave reaches each end of the line.
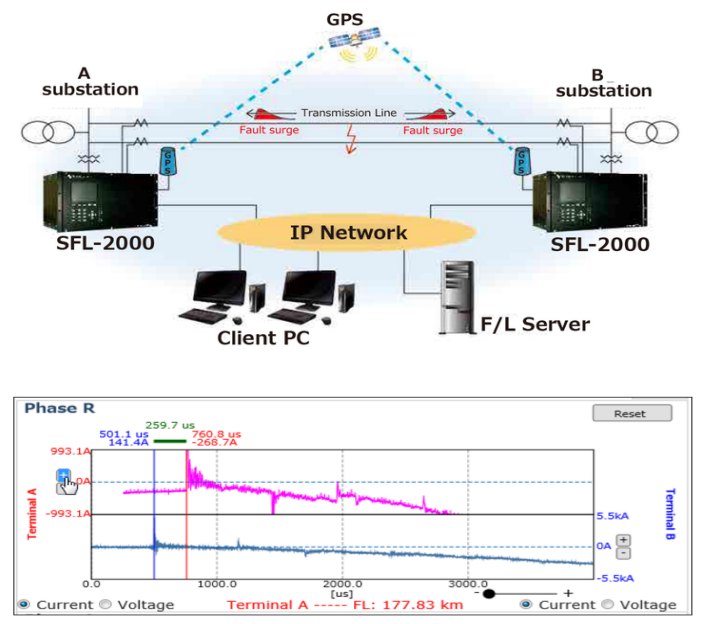

Fig. 1. Traveling wave fault locator SFL-2000 on transmission line

\section{A. Single Ended Method}

Figure 2 for a fault at location $\mathrm{F}$ on a line of length $L=66.9 \mathrm{~km}$ (the Bewley diagram is shown in Figure 14). The fault is $\mathrm{m}(\mathrm{km})$ away from the Hoa Khanh terminal, that is suspected to be in the second half of the line Hoa Khanh-Thanh My. A current TW is launched from the $\mathrm{F}$ at $t_{0}=40 \mathrm{~ms}$ and arrives at the Hoa Khanh terminal at $t_{1 B}$. To discriminate the reflection wave of fault point, the number of samples in the selected window is limited to the interval from $t_{1 B}$ to $40.825 \mathrm{~ms}$ after the fault occurs. Part of the wave transmission travels toward the bus Da Nang and then returns to the Hoa Khanh terminal at $t^{\prime}{ }_{1 A}$. It has opposite polarity with the first traveling wave $\left(t^{\prime}{ }_{1 A^{-}} t_{1 B}=\right.$ constant $)$. So we identify and eliminate false peak due to the effect of this wave. Another part of the wave reflects, travels back toward the fault, reflects back from the fault, and then returns to the Hoa Khanh terminal at $t_{2 B}$. It has larger crest-current wave than the reflected surge from the Thanh My terminal at $t^{\prime}{ }_{1 C}$ with the same polarity. Similarly, in the previously examined case, the fault is assumed to occur at the first half of the line as shown in Figure 13 then the number of samples in the chosen window has limited the interval from $t_{1 B}$ to $40.4125 \mathrm{~ms}$. Now, the distance to the fault location from TWFL B is [2]:

$$
m=\frac{v \times\left(t_{2 B}-t_{1 B}\right)}{2}
$$

Wave propagation velocity is $v=1 / \sqrt{L_{1} \times C_{1}} \approx 243250 \mathrm{~km} / \mathrm{s}$ (where $L_{l}=0.0013 \mathrm{H} / \mathrm{km}$ is the inductance and $C_{I}=0.013 \mu \mathrm{F} / \mathrm{km}$ is the capacitance of the propagation medium). When an external fault behind the Hoa Khanh terminal launches a traveling wave as shown in Figure 3, the TWFL B sees an initial wave behind it with $t_{1 B}=t_{1}$, which travels across the transmission line to the Thanh My terminal and is reflected back to the Hoa Khanh terminal after the known TW line propagation time $\left(t_{L}=0.275 \mathrm{~ms}\right)$ with $t_{2 B}=2 \times t_{L}+t_{1}$. The TWFL B displays $L$.

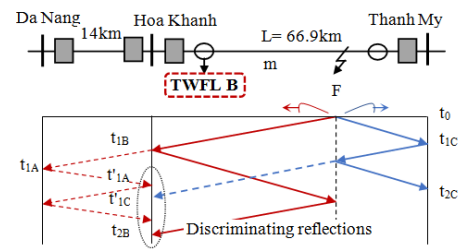

Fig. 2. Single end TWFL with internal fault

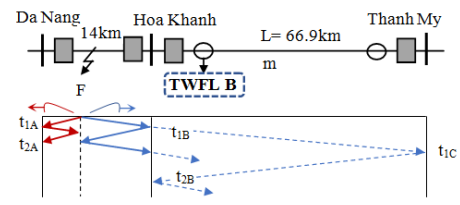

Fig. 3. Single end TWFL with external fault

Review: The single-ended method estimates the accurate fault location of an internal fault and does not require data synchronization. However, the accuracy of this method depends on the accuracy of $L, v$, sampling frequency, and errors in wave detection. If the impulse wave cannot be captured successfully or the impulse wave does not exist at all at the fault occurrence, the fault location will fail. For instance, strong buses on power system network influencing the voltage and current waveforms due to line impedances can reduce the amplitude of voltage waves making them harder to detect, and thus reducing the TWFL accuracy [4].

\section{B. Double Ended Method}

When an internal fault $\mathrm{F}$ occurs at $t_{0}$, waves generated at $\mathrm{F}$ run towards stations Hoa Khanh and Thanh My (Figure 4). The double-ended method determines the arrival time based on the rising point of surge waveform recorded at both end terminals $\left(t_{1 B}\right.$ at the Hoa Khanh, $t_{1 C}$ at the Thanh My) and then locates the fault point from the equation shown below $[2,3]$.

$$
m=\frac{L}{2}+\frac{v \times\left(t_{1 B}-t_{1 C}\right)}{2}=\frac{L+v \times \Delta t}{2}
$$

We can calculate $v$ using the line length $L=66.9 \mathrm{~km}$ and the arrival time of the surge generated by manually closing a circuit breaker at Thanh My terminal as shown in Figure 5 [3]: $v=L /\left(t_{1 B}-t_{1 C}\right)=243270 \mathrm{~km} / \mathrm{s}$. When an external fault behind the 
Hoa Khanh terminal launches a traveling wave, the TWFL B sees an initial wave with either positive or negative polarity with $t_{1 B}=t_{1}$ which travels across the transmission line to the Thanh My terminal. The TWFL $C$ sees the same initial wave $t_{L}=0.275 \mathrm{~ms}$ later with opposite polarity $\left(t_{1 C}=t_{L}+t_{1}\right)$. The distance to the fault location from TWFL B is $L$.

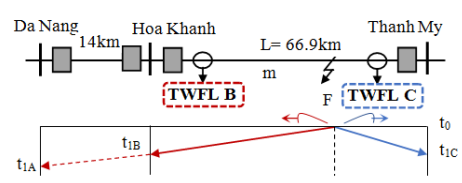

Fig. 4. Double ended TWFL with internal fault

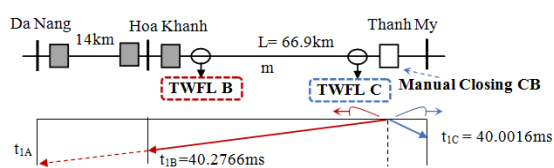

Fig. 5. Calculate propagation velocity

Review: Double-ended method requires data from both terminal ends to be synchronized. It estimates the accurate fault location of an internal fault. The accuracy of this method is effected by communication and precise timing stamp GPS. This method is more expensive than the single-ended method.

\section{POWER SYSTEM UNDER STUDY}

Recorded data from a real system are not available to evaluate the performance of the TWFL. Instead, the power system supplied from both ends can be modeled by Matlab Simulink software (Figure 6). The overhead line Hoa KhanhThanh My is $66.9 \mathrm{~km}$ long, and the system nominal operating voltage is $220 \mathrm{kV}, 50 \mathrm{~Hz}$. This model consists of:

1. The transmission line: three phase section lines are used to represent the distributed parameter transmission line. Line sequence impedance is:

$$
\begin{aligned}
& R_{L 1}=0.07(\Omega / \mathrm{km}), R_{L 0}=0.2164(\Omega / \mathrm{km}) . \\
& L_{L 1}=0.0013(\mathrm{H} / \mathrm{km}), L_{L 0}=0.0044(\mathrm{H} / \mathrm{km}) . \\
& C_{L 1}=0.013(\mu \mathrm{F} / \mathrm{km}), C_{L 0}=0.0085(\mu \mathrm{F} / \mathrm{km}) .
\end{aligned}
$$

2. A load of $220 \mathrm{kV}, 56 \mathrm{MW}$, and $34 \mathrm{kVAR}$ is connected to the bus Hoa Khanh and Thanh My.

3. Three phase fault block to deduce fault types and fault resistance varies from 1 to $35 \mathrm{ohms}$.

4. Three-phase measuring blocks to measure the three phase line and load current and voltage values.

5. A TWFL model is located at Hoa Khanh bus. It has been developed for fault detection, fault classification, fault direction and fault location which will be presented in section IV.

\section{TRAVELING WAVE FAULT LOCATOR MODEL}

This subsection helps to understand how the TWFL works. The simulation model of the TWFL has been designed with six functional flow block diagram (Figure 7) by using Simulink.

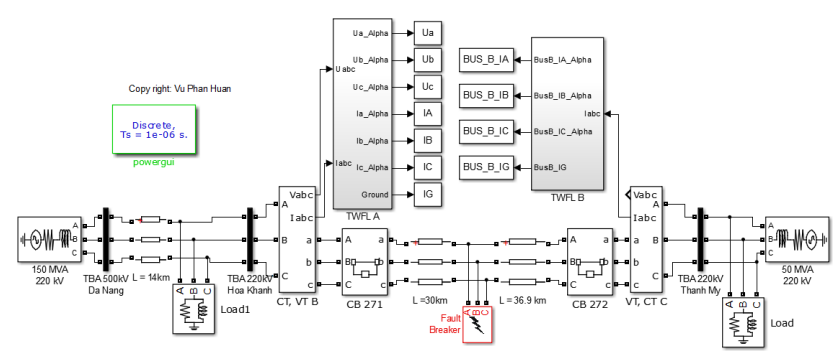

Fig. 6. Power system model

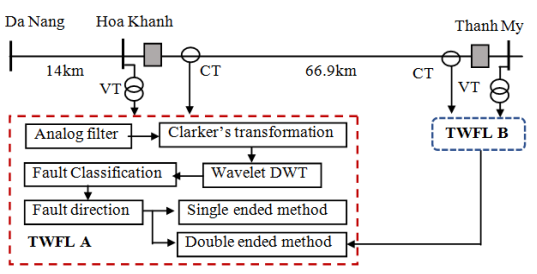

Fig. 7. Flowchart of travelling wave fault locator operation

\section{A. Analog Filter}

A fault generates current and voltage traveling waves that propagate along the overhead line. Most of them contain a significant amount of high-frequency components. The TWFL collects TWs from conventional CT class 5P20, CVT class 3P. Then it uses an analog Chebyshev type II filter to remove the fundamental component $50 \mathrm{~Hz}$ and a high-pass filter with a cutoff frequency of $3 \mathrm{kHz}$ for phase currents and voltages.

\section{B. Clarke's Tranformation}

To reduce the effect of mutual coupling between phases, this paper utilizes the Clarke transformation to convert the three-phase currents and voltages into the $\alpha, \beta$ and 0 mode components. For example, we use three sets of Clarke components with reference to the A-phase, B-phase, and Cphase of current signals, as follows [5]:

$$
\begin{aligned}
& {\left[\begin{array}{c}
I_{A}^{\alpha} \\
I_{A}^{\beta} \\
I_{A}^{0}
\end{array}\right]=\frac{1}{3}\left[\begin{array}{ccc}
2 & -1 & -1 \\
0 & \sqrt{3} & -\sqrt{3} \\
1 & 1 & 1
\end{array}\right]\left[\begin{array}{l}
I_{A} \\
I_{B} \\
I_{C}
\end{array}\right]} \\
& {\left[\begin{array}{c}
I_{B}^{\alpha} \\
I_{B}^{\beta} \\
I_{B}^{0}
\end{array}\right]=\frac{1}{3}\left[\begin{array}{ccc}
-1 & 2 & -1 \\
-\sqrt{3} & 0 & \sqrt{3} \\
1 & 1 & 1
\end{array}\right]\left[\begin{array}{l}
I_{A} \\
I_{B} \\
I_{C}
\end{array}\right]} \\
& {\left[\begin{array}{l}
I_{C}^{\alpha} \\
I_{C}^{\beta} \\
I_{C}^{0}
\end{array}\right]=\frac{1}{3}\left[\begin{array}{ccc}
-1 & -1 & 2 \\
\sqrt{3} & -\sqrt{3} & 0 \\
1 & 1 & 1
\end{array}\right]\left[\begin{array}{l}
I_{A} \\
I_{B} \\
I_{C}
\end{array}\right]}
\end{aligned}
$$

\section{Discrete Wavelet Transform}

This function is developed by using an $\alpha$ mode and 0 (ground) mode component which capture voltages and current with a sampling rate of $10 \mathrm{MHz}$ at TWFL $\mathrm{A}\left(\mathrm{U}_{\mathrm{A}}^{\alpha}, \mathrm{U}^{\alpha}{ }_{\mathrm{B}}, \mathrm{U}^{\alpha}{ }_{\mathrm{C}}, \mathrm{I}_{\mathrm{A}}^{\alpha}\right.$, $\mathrm{I}_{\mathrm{B}}^{\alpha}, \mathrm{I}_{\mathrm{C}}^{\alpha}, \mathrm{I}_{\mathrm{G}}$ ), and TWFL B (Bus_B $\mathrm{I}^{\alpha}{ }_{\mathrm{A}}, \mathrm{Bus}{ }_{-} \mathrm{B} \mathrm{I}_{\mathrm{B}}^{\alpha}$, Bus_B I $^{\alpha}{ }_{\mathrm{C}}$, Bus_B_ $\left.\mathrm{I}_{\mathrm{G}}\right)$. By using DWT Daubechies 4 mother wavelet (db4) 
during the fault surges from the state steady sampled currents and voltages, we can rapidly extract the first level detail Wavelet Coefficients $(\mathrm{Cd} 1)$, frequencies up to $5 \mathrm{MHz}$ which is enough for the TWFL transient frequency. Now it is easy to determine the times of traveling wave occurrences (peaks can be observed on waveform) and to reveal their travel times between the point fault $\mathrm{F}$ and TWFL.

\section{Fault Detection and Fault Classification}

Under normal conditions, Cd1 of phase A, B, C, and Ground are zero (Figure 8). Under fault conditions, if the Cd1 of ground is zero, the fault is identified as an ungrounded fault or as a grounded fault if it is nonzero. The Cd1 of phase A, B, and $\mathrm{C}$ are available for all fault types. Consider the $\mathrm{AG}$ fault occurring on the transmission line at time 40ms (Figure 9). Both reflection and refraction of the $\mathrm{Cd} 1$ of current phase A and ground with the large amplitude occur at 40.0 to $40.8 \mathrm{~ms}$ (or $2.0 \times 10^{5}$ to $2.04 \times 10^{5}$ samples). They are approximately twice as large as the $\mathrm{Cd} 1$ of current phase B, C (healthy phases), and they have opposite polarity. Consider the $\mathrm{BC}$ fault (Figure 10), the $\mathrm{Cd} 1$ of current phase $\mathrm{B}$ and $\mathrm{C}$ are also considerably larger than the $\mathrm{Cd} 1$ in the healthy phase (A, ground), and these faulted phases have opposite polarities. Based on information relationship between the squared $\mathrm{Cd} 1$ magnitude of the first current traveling waves in each phase, the TWFL can make fault type decisions that can be summarized in Table I.

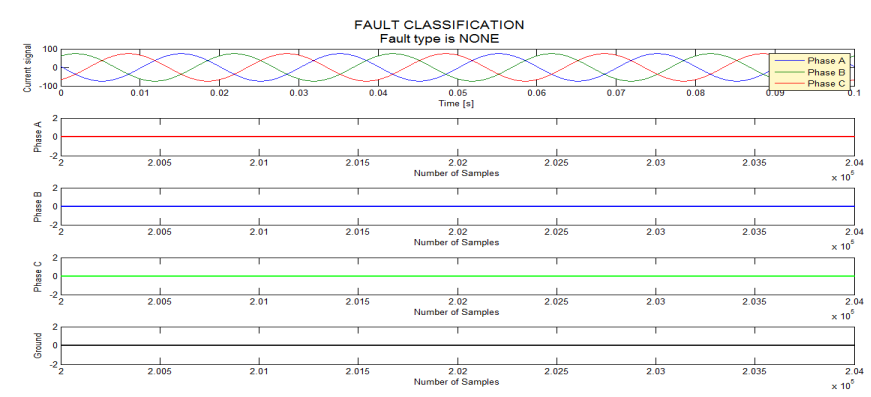

Fig. 8. The $\mathrm{Cd} 1$ of phase $\mathrm{A}, \mathrm{B}, \mathrm{C}$ and ground at normal condition

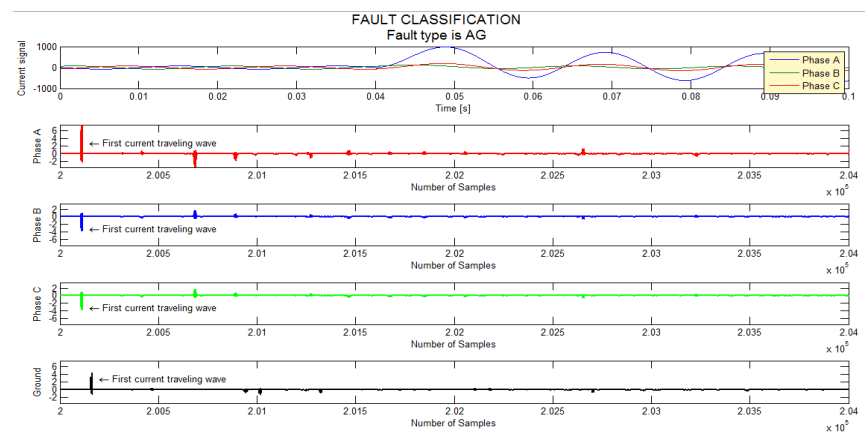

Fig. 9. The $\mathrm{Cd} 1$ of phase $\mathrm{A}, \mathrm{B}, \mathrm{C}$ and ground at $\mathrm{AG}$ fault

\section{E. Direction Fault}

The single-ended method can make a directional decision based on the polarity relationship between the first voltage and current traveling waves. For a fault on the transmission line in the forward direction, the voltage and current traveling waves observed by the relay have opposite polarity (Figure 11). For a fault on the transmission line in the reverse direction, the voltage and current traveling waves observed by the relay have the same polarity as in Figure 12. The double end method compares time-aligned current first TWs at both ends of the protected line. For an external fault, a TW that entered one terminal with a given polarity leaves the other terminal with the opposite polarity exactly after $\Delta \mathrm{t}=\mathrm{t}_{\mathrm{L}}$. For an internal fault, a TW that entered one terminal with a given polarity leaves the other terminal with the same polarity, $\Delta \mathrm{t}<\mathrm{t}_{\mathrm{L}}[2]$.

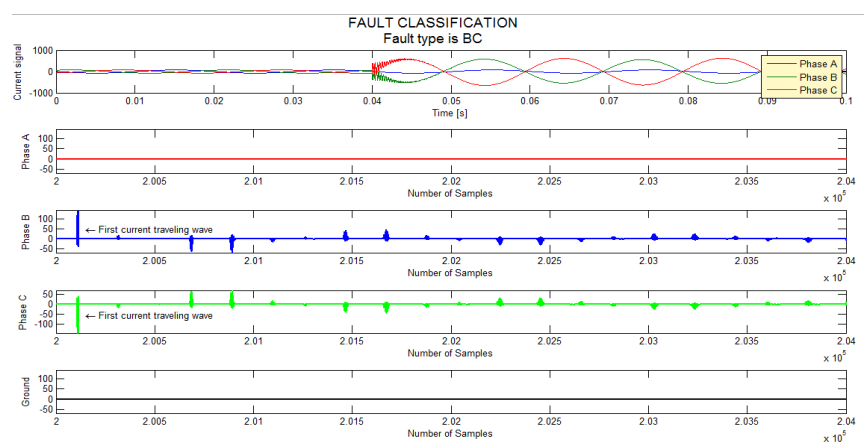

Fig. 10. The Cd1 of phase A, B, C and ground at BC fault

TABLE I. FAULT TYPE CLASSIFICATION

\begin{tabular}{|c|c|c|}
\hline $\begin{array}{l}\text { Fault } \\
\text { Type }\end{array}$ & Condition & $\begin{array}{c}\text { Selection } \\
\text { signal for } \\
\text { fault } \\
\text { location }\end{array}$ \\
\hline AG & $\begin{array}{c}\text { Mag_A/Mag_B }>1.5 \text { and Mag_A/Mag_C }>1.5 \text { and } \\
\text { Mag_G/Mag_A }>0.2\end{array}$ & Cd1_A \\
\hline BG & $\begin{array}{c}\text { Mag_B/Mag_A }>1.5 \text { and Mag_B/Mag_C }>1.5 \text { and } \\
\text { Mag_G/Mag_B }>0.2\end{array}$ & Cd1_B \\
\hline CG & 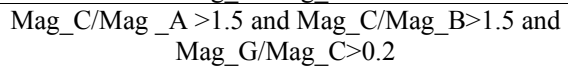 & $\mathrm{Cd} 1 \_\mathrm{C}$ \\
\hline $\mathrm{AB}$ & $\begin{array}{c}\text { Mag_A/Mag_C }>1.5 \text { and Mag_B/Mag_C }>1.5 \text { and } \\
\text { Mag_G/Mag_A }<0.2 \text { and Mag_G/Mag_B }<0.2\end{array}$ & $\begin{array}{l}\text { Cd1_A or } \\
\text { Cd1_B }\end{array}$ \\
\hline $\mathrm{BC}$ & $\begin{array}{c}\text { Mag_B/Mag_A }>1.5 \text { and Mag_C/Mag_A }>1.5 \text { and } \\
\text { Mag_G/Mag_B }<0.2 \text { and Mag_G/Mag_C }<0.2\end{array}$ & $\begin{array}{c}\text { Cd1_B or } \\
\text { Cd1_C }\end{array}$ \\
\hline $\mathrm{CA}$ & $\begin{array}{c}\text { Mag_C/Mag_B }>1.5 \text { and Mag_A/Mag_B }>1.5 \text { and } \\
\text { Mag_G/Mag_C }<0.2 \text { and Mag_G/Mag_A }<0.2\end{array}$ & $\begin{array}{l}\text { Cd1_ } \mathrm{C} \text { or } \\
\text { Cd1_A }\end{array}$ \\
\hline ABG & $\begin{array}{l}\text { Mag_A/Mag_C }>1.5 \text { and Mag_B/Mag_C }>1.5 \text { and } \\
\text { Mag_G/Mag_A }>0.2 \text { and Mag_G/Mag B }>0.2\end{array}$ & $\begin{array}{l}\text { Cd1_A or } \\
\text { Cd1 B }\end{array}$ \\
\hline BCG & $\begin{array}{c}\text { Mag_B/Mag_A }>1.5 \text { and Mag_C/Mag_A }>1.5 \text { and } \\
\text { Mag_G/Mag_B }>0.2 \text { and Mag_G/Mag_C }>0.2\end{array}$ & $\begin{array}{c}\text { Cd1_B or } \\
\text { Cd1_C }\end{array}$ \\
\hline CAG & $\begin{array}{c}\text { Mag_C/Mag_B }>1.5 \text { and Mag_A/Mag_B }>1.5 \text { and } \\
\text { Mag_G/Mag_C }>0.2 \text { and Mag_G/Mag_A }>0.2\end{array}$ & $\begin{array}{l}\text { Cd1_ } \mathrm{C} \text { or } \\
\text { Cd1_A }\end{array}$ \\
\hline $\mathrm{ABC}$ & Mag_A $>1.5$ and Mag_B $>1.5$ and Mag_C $>1.5$ & $\begin{array}{c}\mathrm{Cd} 1 \_\mathrm{A} \text { or } \\
\mathrm{Cd}{ }^{-} \mathrm{B} \text { or } \\
\mathrm{Cd} 1{ }_{C}\end{array}$ \\
\hline
\end{tabular}

\section{F. Distance Calculations}

Because the frequency response for CTs is better than for CCVTs [4], we chose to use current signals for fault location. In the case when TWFL identifies a forward or internal fault, both single end and double end methods are activated. We use Matlab's find peaks function to find values and locations of Cd1 maxima in a time period set according to the travel time of the line. The fault distance is given by (1) and (2). 

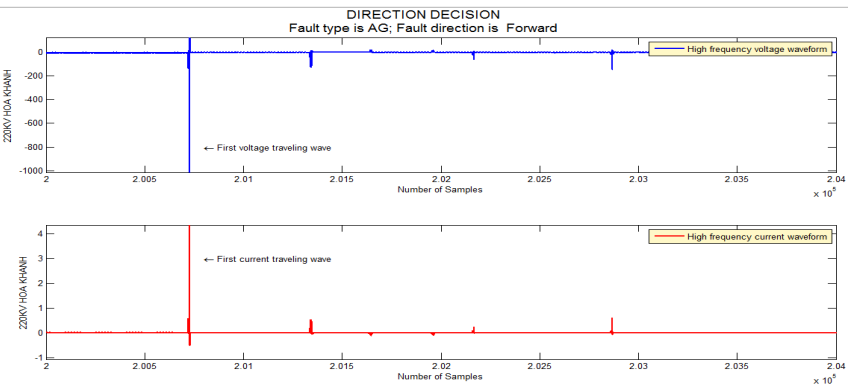

Fig. 11. Curent and voltage polarities of phase $\mathrm{A}$ in fault forward direction
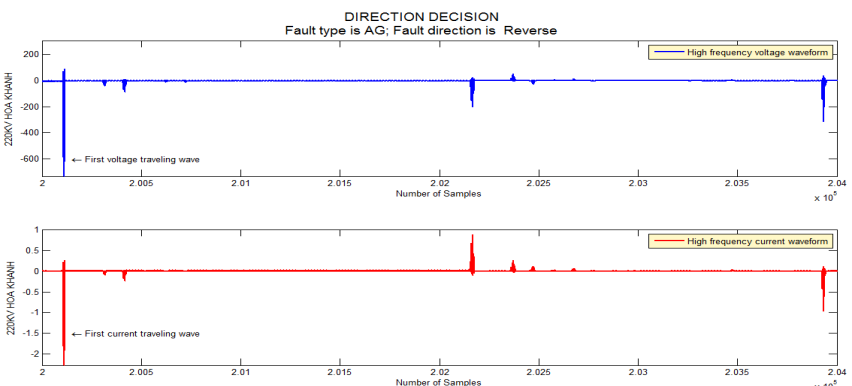

Fig. 12. Curent and voltage polarities of phase $\mathrm{A}$ in fault reverse direction

\section{SIMULATION RESULTS}

After building, the proposed model is ready to analyze the operation of TWFL applied with variation in fault parameters such as fault type, fault location (from $-5 \mathrm{~km}$ to $65 \mathrm{~km}$ ), fault resistance from $1 \Omega$ to $35 \Omega$ (increasing the fault resistance seen at relay point as though moving away from the transmission line). The fault creation time is $t_{0}=40 \mathrm{~ms}$. Figure 13 shows the phase currents captured at both terminals and a Beley diagram for a BG fault with $R_{F}=10 \Omega$ that is assumed to occur at a distance of $20 \mathrm{~km}$ from Hoa Khanh bus (forward direction). TWFL B calculates the fault location by the single-ended method with $t_{1 B}=40.0836 \mathrm{~ms}, t_{2 B}=40.2476 \mathrm{~ms}$, due to the traveling time taken by the fault to appear at the TWFL B. Based on the measured TW arrival times, an estimated from (1) fault location $19.9523 \mathrm{~km}$ from the Hoa Khanh terminal emerged. In Figure 14, results are shown where a BCG fault with $R_{F}=25 \Omega$ at a distance of $45 \mathrm{~km}$ from Hoa Khanh bus (forward direction). According to the traveling time taken by the fault to appear at the point $t_{1 B}=40.1866 \mathrm{~ms}, t_{2 B}=40.5566 \mathrm{~ms}$. The TWFL B also uses the single-ended method to calculate fault location is $45.0016 \mathrm{~km}$.

The double-end method's (Figure 15) performance is simpler than the single-ended one. In the case an $\mathrm{AB}$ fault occurs on the transmission line with $R_{F}=15 \Omega$ at $10 \mathrm{~km}$, it calculates the distance to fault based on time tags of traveling wave records acquired at both ends of the faulty line. The first peak of Hoa Khanh terminal occurs at $t_{1 B}=40.0426 \mathrm{~ms}$, the first peak of Thanh My terminal occurs at $t_{1 C}=40.2356 \mathrm{~ms}$, so the distance to the fault point $F$ of TWFL B estimate from (2) is $9.9762 \mathrm{~km}$. As shown in Figure 16, an ABC fault occurs on the transmission line at $35 \mathrm{~km}, R_{F}=15 \Omega$. According to the change in current waveforms during the fault, TWFL B captured point $t_{1 B}=40.1456 \mathrm{~s}$, TWFL C captured $t_{1 C}=40.1326 \mathrm{~s}$, thus the doubleend method of TWFL B estimate is $35.0313 \mathrm{~km}$. Some of the results for various fault cases are given in Table II respectively for $\mathrm{BG}, \mathrm{AB}, \mathrm{BCG}$, and $\mathrm{ABC}$.
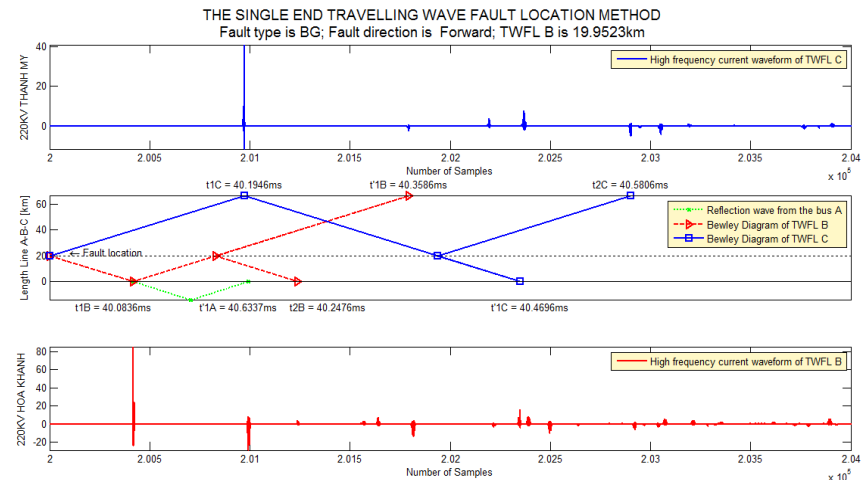

Fig. 13. Phase current waves and Bewley diagram explaining single ended method for a BG fault at $20 \mathrm{~km}$ from the Hoa Khanh terminal

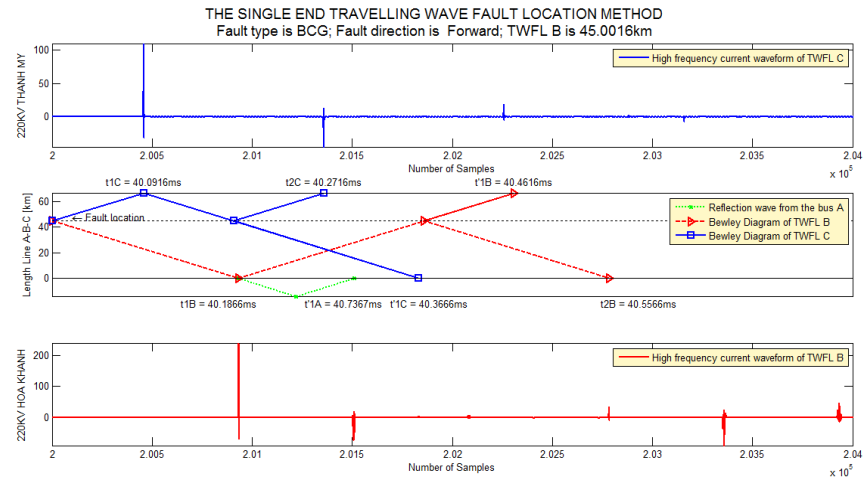

Fig. 14. Phase current waves and Bewley diagram explaining single ended mehtod for a BCG fault at $45 \mathrm{~km}$ from the Hoa Khanh terminal

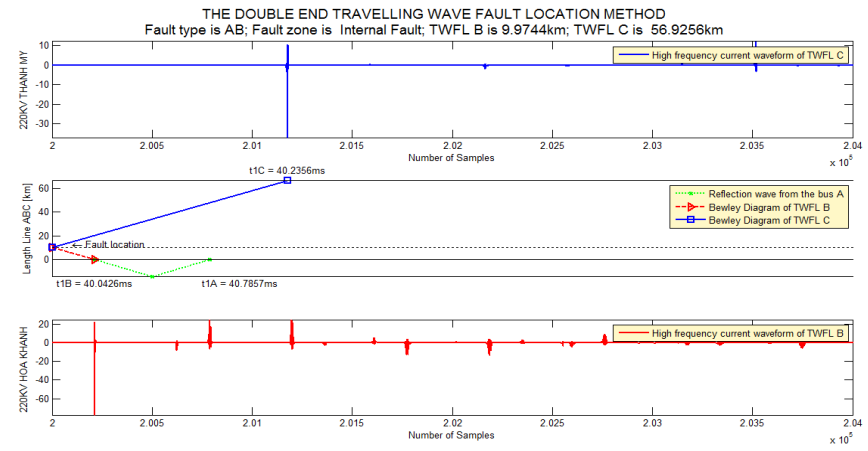

Fig. 15. Phase current waves and Bewley diagram explaining double ended method for $\mathrm{AB}$ fault at $10 \mathrm{~km}$ from the Hoa Khanh terminal

Review: The simulation results show that single ended method locates faults with accuracy less than $\pm 114 \mathrm{~m}$ and double ended method locates faults with accuracy less than $\pm 112 \mathrm{~m}$. 

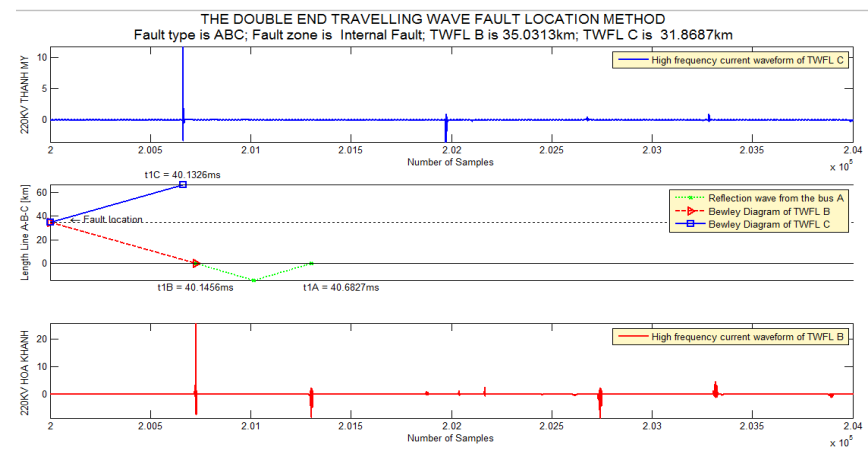

Fig. 16. Phase current waves and Bewley diagram explaining double ended method for ABC fault at $35 \mathrm{~km}$ from the Hoa Khanh terminal

TABLE II. TEST RESULTS OF FAULTS ON TRANSMISSION LINE

\begin{tabular}{|c|c|c|c|c|c|c|}
\hline \multirow{2}{*}{ Phase } & \multirow{2}{*}{$\begin{array}{c}\text { Actual } \\
\text { fault } \\
\text { location } \\
\text { [km] }\end{array}$} & \multirow{2}{*}{$\begin{array}{c}\text { Fault } \\
\text { resistance } \\
{[\Omega]}\end{array}$} & \multicolumn{2}{|c|}{$\begin{array}{l}\text { Single end Method } \\
\text { Estimated Distance } \\
\end{array}$} & \multicolumn{2}{|c|}{$\begin{array}{l}\text { Double end Method } \\
\text { Estimated Distance } \\
\end{array}$} \\
\hline & & & $m[\mathbf{k m}]$ & $\begin{array}{c}\text { Difference } \\
{[\mathrm{m}]}\end{array}$ & $m[\mathrm{~km}]$ & $\begin{array}{c}\text { Difference } \\
{[\mathrm{m}]}\end{array}$ \\
\hline \multirow{7}{*}{ BG } & -5 & 1 & 1 & & 1 & \\
\hline & 10 & 5 & 9.9733 & -26.7 & 9.9762 & -23.8 \\
\hline & 20 & 10 & 19.9523 & -53.3 & 19.9495 & -50.5 \\
\hline & 30 & 15 & 30.0416 & 41.6 & 29.9228 & -77.2 \\
\hline & 40 & 20 & 40.015 & 15 & 39.967 & -33 \\
\hline & 50 & 25 & 49.9883 & -11.7 & 50.112 & 112 \\
\hline & 60 & 30 & 60.889 & 88.9 & 60.0859 & 85.9 \\
\hline \multirow{7}{*}{$\mathrm{AB}$} & -5 & 1 & 1 & & / & \\
\hline & 10 & 5 & 9.9733 & -26.7 & 9.9744 & -25.6 \\
\hline & 20 & 10 & 19.9467 & -53.3 & 19.9495 & -50.5 \\
\hline & 30 & 15 & 30.0416 & 41.6 & 29.9228 & -77.2 \\
\hline & 40 & 20 & 40.015 & 15 & 39.967 & -33 \\
\hline & 50 & 25 & 49.9883 & -11.7 & 50.112 & 112 \\
\hline & 60 & 30 & 59.9616 & -38.4 & 60.0861 & 86.1 \\
\hline \multirow{8}{*}{ BCG } & -5 & 1 & 1 & & 1 & \\
\hline & 5 & 5 & 5.114 & 114 & 5.109 & 109 \\
\hline & 15 & 10 & 14.960 & -40 & 15.0831 & 83.1 \\
\hline & 25 & 15 & 25.055 & 55 & 25.0578 & 57.8 \\
\hline & 35 & 20 & 35.028 & 28 & 35.031 & 31 \\
\hline & 45 & 25 & 45.016 & 16 & 45.0045 & 4.5 \\
\hline & 55 & 30 & 54.932 & -68 & 54.9778 & -22.2 \\
\hline & 65 & 35 & 64.9053 & -94.7 & 64.9512 & -48.8 \\
\hline \multirow{8}{*}{$\mathrm{ABC}$} & -5 & 1 & / & & 1 & \\
\hline & 5 & 5 & 4.9867 & -13.3 & 5.109 & 109 \\
\hline & 15 & 10 & 14.96 & -40 & 15.0831 & 83.1 \\
\hline & 25 & 15 & 25.055 & 55 & 25.0578 & 57.8 \\
\hline & 35 & 20 & 35.0283 & 28.3 & 35.0313 & 31.3 \\
\hline & 45 & 25 & 45.0073 & 7.3 & 45.0045 & 4.5 \\
\hline & 55 & 30 & 54.9806 & -19.4 & 54.9778 & -22.2 \\
\hline & 65 & 35 & 64.954 & -46 & 64.9535 & -46.5 \\
\hline
\end{tabular}

\section{CONCLUSIONS}

Performance evaluation of TWFL systems has become an increasingly important issue given its design, manufacturing, sale/purchase, use, upgrade, tuning, etc. In this study, a TWFL model built into a $220 \mathrm{kV}$ transmission line that can be easily and reliably simulated on the Matlab Simulink software with $10 \mathrm{MHz}$ sampling frequency. The proposed model determines exactly fault types, fault direction and fault location. According to the obtained results, it has been shown that TWFL is more accurate than the traditional impedance-based methods in relay protection. Double-ended method is more accurate than singleended method. Furthermore, the paper demonstrates a Bewley diagram with the times of the current impulse wave at each terminal and results which are intended to help the operator transition from beginner to experienced professionals. This can assist in the research to eliminate factors of misoperation, contribute substantially to the safe, reliable, economical operation and maintenance of overhead transmission lines.

\section{ACKNOWLEDGMENT}

Authors would like to thank Power Transmission Company No. 2, Viet Nam for allowing the use of fault location equipment for $220 \mathrm{kV}$ and $500 \mathrm{kV}$ transmission lines used in this study.

\section{REFERENCES}

[1] K. H. Le, P. H. Vu, “A Studying Of Single Ended Fault Locator On SEL Relay", IETEC'13 Conference, Ho Chi Minh City, Vietnam, December 4-6, 2013

[2] SEL, SEL T400L Ultra high speed transmission line relay traveling wave fault locator high resolution event recorder Instruction Manual, 2018

[3] Kinkei System Corporation, Surge type fault locator system specifications SFL 2000, 2016.

[4] S. Parmar, Fault Location Algorithms for Electrical Power Transmission Lines Methodology, Design, and Testing, MSc Thesis, Delft University of Technology, 2015

[5] B. Kasztenny, A. Guzmán, N. Fischer, M. V. Mynam, D. Taylor, "Practical setting considerations for protective relays that use incremental quantities and traveling waves", 43rd Annual Western Protective Relay Conference, Washington, USA, October 18-20, 2016 